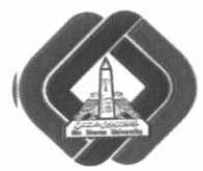

\title{
A PROPOSED METHOD FOR INCREASE ACCURACY OF CLASSIFICATION P300 SPELLER
}

\author{
A. M. Khalaf \\ M. A. El-Desouky \\ M. Z. Rashad \\ qaderneno@gmail.com \\ Faculty of Computers and Information, Mansoura University, Egyp
}

Abstract: A P300 speller is one of applications the brain computer interface (BCI), introduced by Farwell and Donchin in 1988 [1]. In this paper proposed a new method for increase the accuracy of classification P300 speller. Uses a dataset for (16) healthy subjects. The new method including is feature extraction using Principle Component Analysis (PCA), and the classification using Support Vector Machine Linear (SVML). As it was calculated performance and activity of each electrode whether correlated or uncorrelated of speller task. Show that the proposed method is accurate and efficient.

Keywords: Brain Computer Interface (BCI), P300 speller, Accuracy of classification P300.

\section{Introduction}

A brain-computer interface $(\mathrm{BCI})$ sometimes called a mind-machine interface (MMI), direct neural interface (DNI), synthetic telepathy interface (STI) or a brain-machine interface (BMI), is a system that connects the brain to a computer or any other devices directly and avoids the need for peripheral nerve and muscle activities to execute user's actions, measured by brain activity [2]. The main objective of $\mathrm{BCI}$ research is to allow patients with severe motor disabilities to communicate and the ability to control [3].

The challenges of BCI to the highly difficult and complex, noisy, and changes normal of brain signals, chiefly with non-invasive recordings using scalp EEG, in sometimes the computer or other devices misinterprets the brain signals and makes a choice that does not match with user's intention [4].

\subsection{P300 Speller:}

Is a model developed to restore communication in locked-in patients [5]. Signal's P300 is an EEG positive deflection that happens approximately $300 \mathrm{~ms}$ after stimulus and is recorded by centro-parietal passive EEG sensors [6]. Using to select items displayed on a computer screen [7]. All items are displayed and the user focuses his attention (and gaze) onto the target item. Groups of items are successively and random repeatedly flashed, but only the group that contains the target item wills a P300 response. [8]. 
This interface is a $6 \times 6$ matrix that is displayed on the screen, made up of 26 letters of the alphabet, nine numbers and a symbol that enables the cancellation of the previous selection, allows the user to write a text on the computer screen, shown P300 speller in Figure 1 [5].

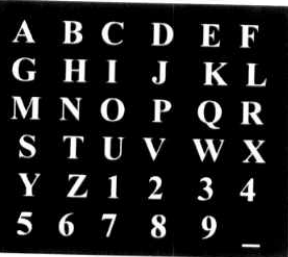

Figure 1: P300 speller matrix [5]

\subsection{Related work:}

In 2012 Perrin Margaux, et al., [8], proposed implemented a P300, including error detection, data recorded in 16 healthy volunteers. Whenever an error was detected, a new decision was made based on a classifier. Deepesh Kumar [9], in transforms (DWT) for feature [9], in 2013, proposed detect the P300 wave. Used discrete wavelet transforms (DWT) for feature extraction of EEG signal, for two subjects. Principal component analysis vector machine (SVM) and artificial of the dimension of the feature, and classification used support SVM classifier better al. [10], propos better performance compared to the method with ANN. In 2014 Benjamin Blankertz et error potential from multichannel false positives, which would classify signals, by designing classifiers that are capable of bounding improvement over the choice of a errors, this method provides a substantial detection of error potentials, Vanitha Namplitude threshold criterion, as it had used for single trial for P300. Brain Computer Interface (BCI) with P300 [11], in 2015, proposed a classification algorithm patients to spell words with the help patients to spell words with the help of their brain signal activities. The proposed model can be used to fast way.

\section{Overview:}

This paper is organised as follows: section 2 is background on BCI, P300 speller and related work, and section 3 the Architecture of BCI systems. Section 4 the proposed method, section 5 the results and discussion, and section 6 future work and conclusion the paper.

\section{Architecture of BCI systems:} $\mathrm{BCI}$ is a brand new branch of pattern recognition. The general architecture of BCI systems is shown in
Figure 2 [12]. 


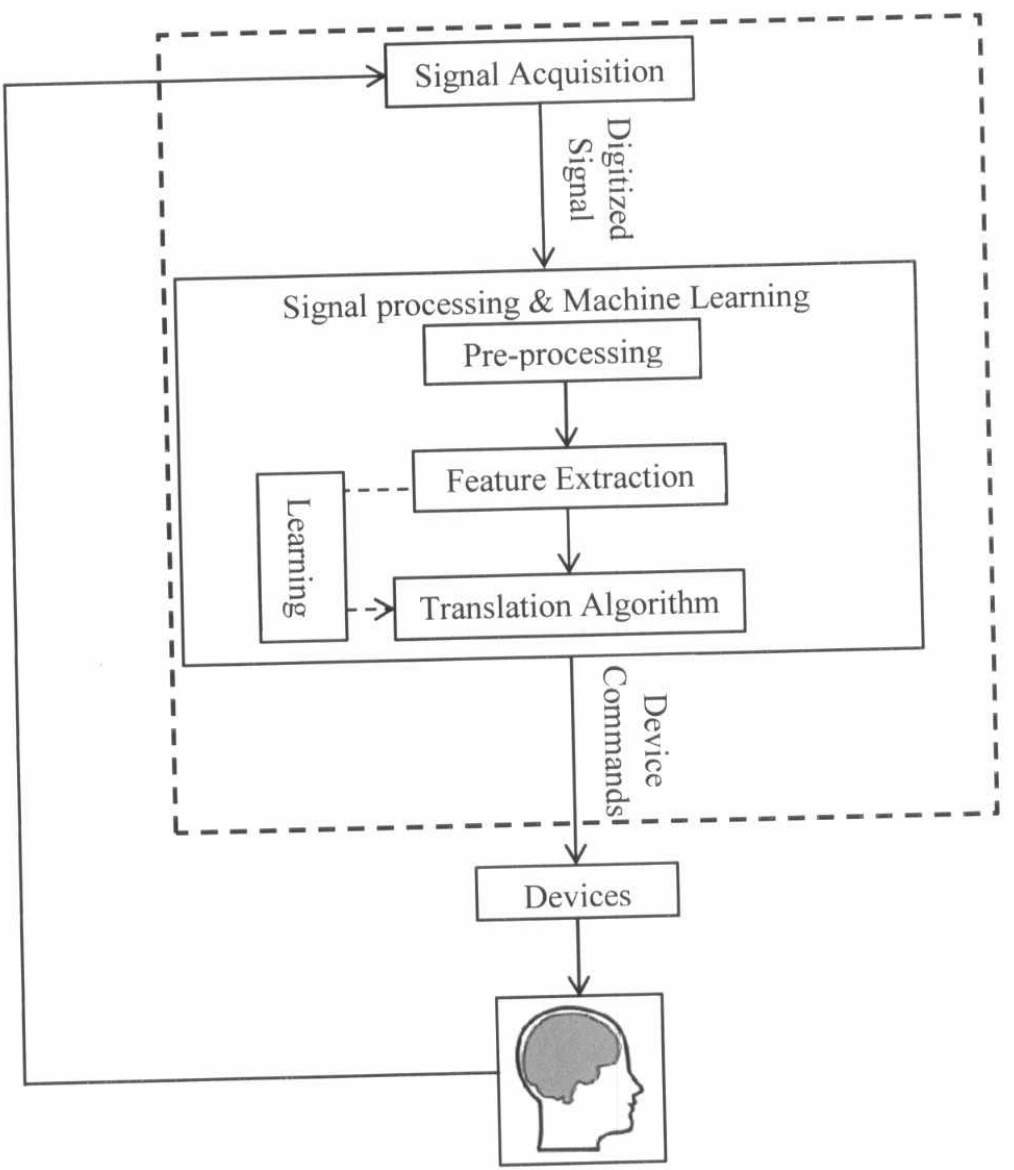

Figure 2: Architecture of BCI systems [12]

4. The proposed method: including four phases, shown in Figure 3.

\subsection{Data description:}

In this paper uses dataset (offline) from web site https:/www.kaggle.com/c/inria-bci-challenge/data, the following data description:

The dataset recorded for sixteen healthy (16 subjects) in the study, (mean age $=28.2$, range 20-37), brain activity was recorded with 56 passive EEG sensors its: (Fp1, Fp2, AF7, AF3, AF4, AF8, F7, F5, F3, F1, Fz, F2, F4, F6, F8, FT7, FC5, FC3, FC1, FCz, FC2, FC4, FC6, FT8, T7, C5, C3, C1, Cz, C2, C4, C6, T8, TP7, CP5, CP3, CP1, CPz, CP2, CP4, CP6, TP8, P7, P5, P3, P1, Pz, P2, P4, P6, P8, PO7, POz, $\mathrm{P} 08, \mathrm{O} 1, \mathrm{O} 2)$, whose placement followed the extended 10-20 system, Eye movements are detected by Electrooculography EOG (1 channel) derivation.

EEG samples recorded from the (56) passive called it's the same sensors (such as: Fpl EEG samples recorded from Fp1) and other. 


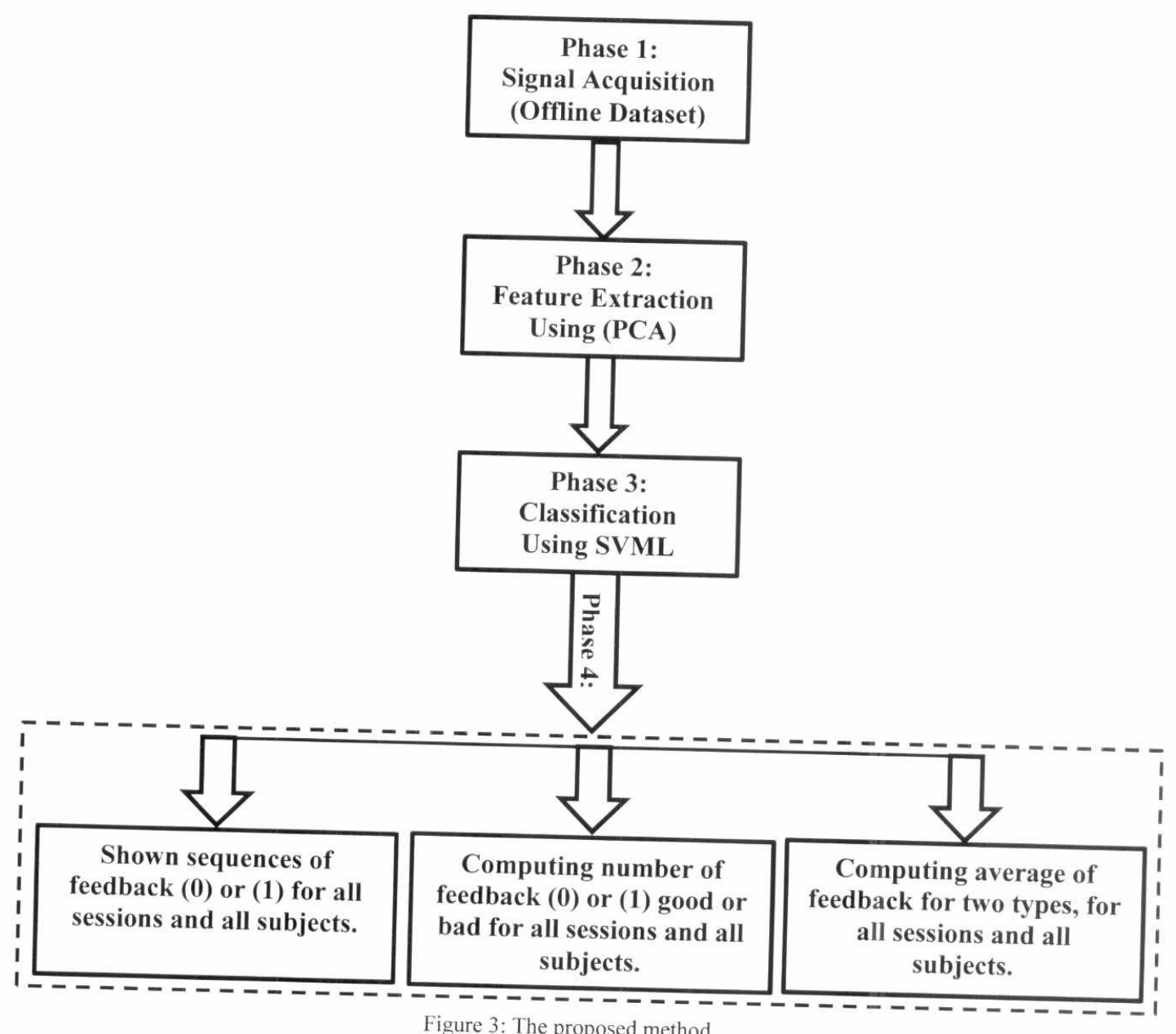

fifth (last session) one for which (5) sessions, (60) feedbacks were provided in each session except the one for which (100) feedbacks were provided.

The subjects had to go through five copy spelling sessions. Each session consisted of twelve 5-letter words, except the fifth which consisted of twenty 5-letter words.

\subsection{Feature extraction:}

In this stage, using Principle Component Analysis (PCA) is a statistical features extraction method that uses a linear transformation to convert a set of observations possibly correlated into a set of components from the input principal components. Linear transformation generates a set of component has the highest possible variance. This to their variance in such a way that the first principal different components. 
Used (PCA) on the Dataset, for feature extraction based on percentage for bad and good feedback, the ercentage for good feedback (in.side mean to target), the percentage for bad feedback (out.side mean to out target) for all sessions and all subjects, (340) feedback for each session ( 240 feedback for the first four sessions, 100 feedback for fifth (last) session)), Bad feedback (negative or 0 ) is when the selected item is different from the expected item. Good feedback (positive or 1) is when the selected item is similar to the expected item.

The following equation computing the percentages for bad or good feedback:

$$
\begin{aligned}
& \frac{N}{60}=\frac{X}{100} \\
& N * 100=X^{* 60} \\
X= & \frac{N * 100}{60}
\end{aligned}
$$

Where $N$ (number of feedback whether good 1 or bad 0 for the first four sessions), $X$ (the percentage of for two types), as for the fifth session (last), it was a (100) feedback and this is calculated ratio percentage directly.

During a P-300 trial users are presented with letters and numbers and are tasked with spelling words. Using electroencephalogram (EEG) data the speller attempts to determine which letter the user is thinking of and presents him/her with this letter. If the letter coincides with the same letter the user was thinking about then the feedback is regarded as positive, otherwise the feedback is negative. The challenge is to predict, based on the user's response to the feedback event, whether feedback was positive ( 1 good) or negative $(0 \mathrm{bad})$.

After error trials the error related potentials (ERP) characterized two items: negative wave denoted by (NE), positive wave denoted by (PE) [13]. After feedback whether good or bad, it's a place an event the error.

\subsection{Classification:}

In this stage using Support Vector Machine Linear (SVML) is a classifier constructs a hyperplane or set of hyperplanes, in order to separate the feature vectors into binary or multiclasses, such a classifier is regarded as a linear classifier, since it uses one or several hyperplanes, but in this case classification into two classes (in.side) and (out.side) based on percentage its obtained from feedback (0 or 1$)$. And SVM calculated number of feedback whether ( 0 bad (negative) or 1 good (positive)) for all subjects and all sessions. Finally explain sequences of feedback for two types, for all subjects and all sessions.

The following parameters measure (the percent in the results and discussions section):

(a) Accuracy $=(\mathrm{TP}+\mathrm{TN}) /(\mathrm{TP}+\mathrm{TN}+\mathrm{FP}+\mathrm{FN})$ activity of the classifier.

(b) Sensitivity $=\mathrm{TP} /(\mathrm{TP}+\mathrm{FN})$ the ability to errors detection properly.

(c) Specificity $=\mathrm{TN} /(\mathrm{TN}+\mathrm{FP})$ the ability to trials detection properly.

(d) Precision=TP/(TP+FP) The percentage of good feedback (positive or 1)

TP: true positive, TN: true negative, FP: false positive, FN: false negative. 


\section{Results and Discussion:}

The accuracy, sensitivity, specificity, and precision of classification its $(90 \%, 80 \%, 85 \%$ and $71 \%$ respectively), after applied (PCA) for feature extraction and (SVML) for classification appear the
results following:

Table 1: Explain the percentage for feedback ( 0 and 1) for (1 \& 2 of sessions).

\begin{tabular}{|c|c|c|c|c|}
\hline \multirow{2}{*}{ Subjects } & \multicolumn{2}{|c|}{ Session 1 for 16 subjects } & \multicolumn{2}{c|}{ Session 2 for 16 subjects } \\
\cline { 2 - 5 } & Out.side (\%) & In.side (\%) & Out.side (\%) & In.side (\%) \\
\hline $\mathbf{1}$ & 16.66 & 83.33 & 30 & 70 \\
\hline $\mathbf{2}$ & 1.66 & 98.33 & 5 & 95 \\
\hline $\mathbf{3}$ & 6.66 & 93.33 & 11.66 & 88.33 \\
\hline $\mathbf{4}$ & 23.33 & 76.66 & 30 & 70 \\
\hline $\mathbf{5}$ & 31.66 & 68.33 & 31.66 & 68.33 \\
\hline $\mathbf{6}$ & 36.66 & 63.33 & 50 & 50 \\
\hline $\mathbf{7}$ & 38.33 & 61.66 & 26.66 & 73.33 \\
\hline $\mathbf{8}$ & 26.66 & 73.33 & 30 & 70 \\
\hline $\mathbf{9}$ & 10 & 90 & 28.33 & 71.66 \\
\hline $\mathbf{1 0}$ & 15 & 85 & 23.33 & 76.66 \\
\hline $\mathbf{1 1}$ & 28.33 & 71.66 & 23.33 & 76.66 \\
\hline $\mathbf{1 2}$ & 3.33 & 96.66 & 3.33 & 96.66 \\
\hline $\mathbf{1 3}$ & 5 & 95 & 6.66 & 93.33 \\
\hline $\mathbf{1 4}$ & 30 & 70 & 40 & 60 \\
\hline $\mathbf{1 5}$ & 10 & 90 & 15 & 85 \\
\hline $\mathbf{1 6}$ & 41.66 & 58.33 & 38.33 & 61.66 \\
\hline Average & $\mathbf{2 0 . 3 0 \pm 0 . 0 2}$ & $\mathbf{7 9 . 6 8} \pm \mathbf{0 . 0 2}$ & $\mathbf{2 4 . 5 8} \mathbf{0 . 0 1}$ & $\mathbf{7 5 . 4 1} \pm \mathbf{0 . 0 1}$ \\
\hline
\end{tabular}

The percentage for good feedback (1) present in.side (to target), the percentage for bad feedback $(0)$ present out.side (out target), note from table (1), the percentage and average for good feedback (1) high 0.01 in session 2).

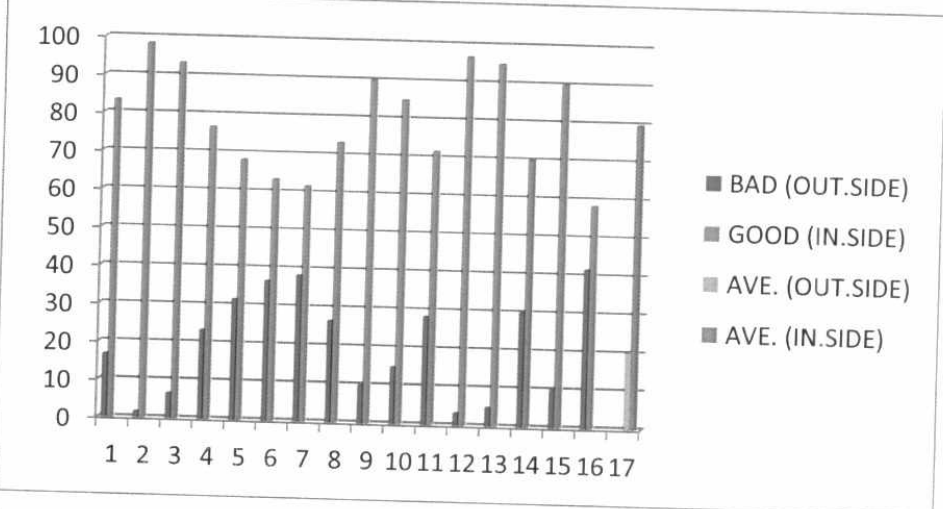

Figure 4: the chart table explains in.side, out.side, average in.side, and average out.side for session 1. 


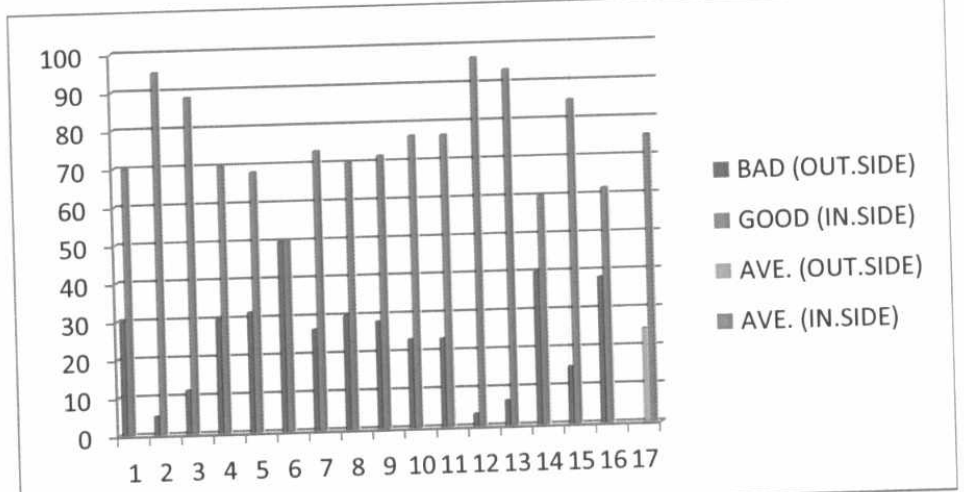

able explains in.side, out.side, average in.side, and average out.side for session 2 , note the subject (6) percentage for feedback ( 0 and 1$)$ its equal.

Table 2: Explain the percentage for feedback ( 0 and 1$)$ for ( $3 \& 4$ of sessions).

\begin{tabular}{|c|c|c|c|c|}
\hline \multirow{2}{*}{ Subjects } & \multicolumn{2}{|c|}{ Session 3 for 16 subjects } & \multicolumn{2}{c|}{ Session 4 for 16 subjects } \\
\cline { 2 - 5 } & Out.side (\%) & In.side (\%) & Out.side (\%) & In.side (\%) \\
\hline $\mathbf{1}$ & 40 & 60 & 41.66 & 58.33 \\
\hline $\mathbf{2}$ & 8.33 & 91.66 & 6.66 & 93.33 \\
\hline $\mathbf{3}$ & 5 & 95 & 11.66 & 88.33 \\
\hline $\mathbf{4}$ & 30 & 70 & 35 & 65 \\
\hline $\mathbf{5}$ & 45 & 55 & 45 & 55 \\
\hline $\mathbf{6}$ & 60 & 40 & 41.66 & 58.33 \\
\hline $\mathbf{7}$ & 30 & 70 & 30 & 70 \\
\hline $\mathbf{8}$ & 36.66 & 63.33 & 41.66 & 58.33 \\
\hline $\mathbf{9}$ & 40 & 60 & 43.33 & 56.66 \\
\hline $\mathbf{1 0}$ & 23.33 & 76.66 & 25 & 75 \\
\hline $\mathbf{1 1}$ & 30 & 70 & 31.66 & 68.33 \\
\hline $\mathbf{1 2}$ & 5 & 95 & 8.33 & 91.66 \\
\hline $\mathbf{1 3}$ & 10 & 90 & 8.33 & 91.66 \\
\hline $\mathbf{1 4}$ & 35 & 65 & 28.33 & 71.66 \\
\hline $\mathbf{1 5}$ & 23.33 & 76.66 & 38.33 & 61.66 \\
\hline $\mathbf{1 6}$ & 50 & 50 & 35 & 65 \\
\hline Average & $\mathbf{2 9 . 4 7 \pm 0 . 0 2}$ & $\mathbf{7 0 . 5 1 \pm 0 . 0 2}$ & $\mathbf{2 9 . 4 7 \pm 0 . 0 2}$ & $\mathbf{7 0 . 5 1 \pm 0 . 0 2}$ \\
\hline & & & & \\
\hline & & & & \\
\hline
\end{tabular}

The percentage for good feedback (1) present in.side (to target), the percentage for bad feedback $(0)$ (arget), note from table (2), the percentage and average for good feedback (1) high compare with percentage and average for bad feedback $(0)$, with an error ratio its $(0.02$ in sessions $3 \&$ 4 respectively). 


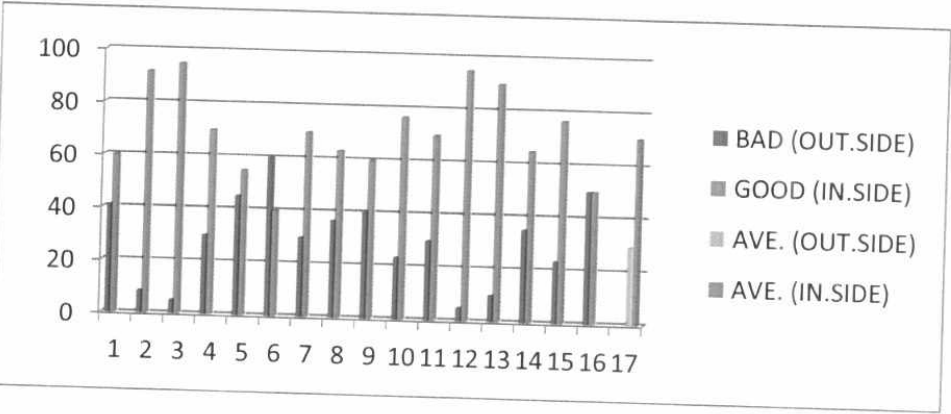

Figure 6: the chart table explains in.side, out.side, average in.side, and average out.side for session 3 , note the subject (6) the
percentage of percentage of $\mathrm{b} 100 \ldots 1)$ its equal.

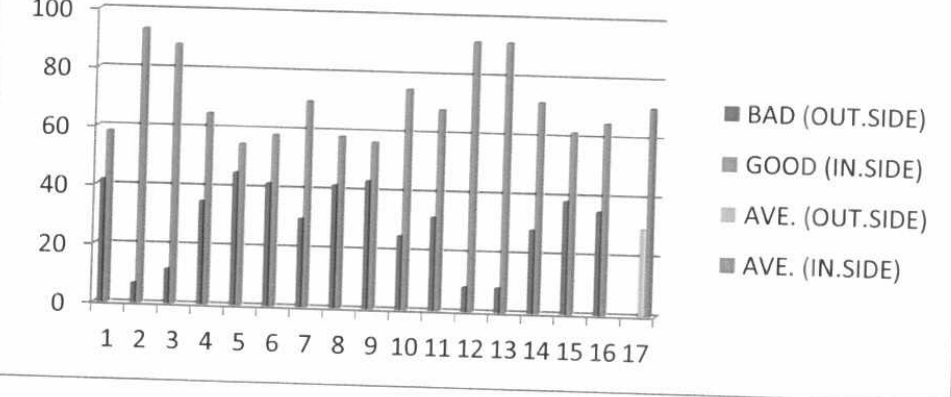

Figure 7: the chart table explains in.side, out.side, average in.side, and average out.side for session 4 .

Table 3: Explain the percentage for feedback (0 and 1) for (session 5).

\begin{tabular}{|c|c|c|}
\hline \multirow[b]{2}{*}{ Subjects } & \multicolumn{2}{|c|}{ Sessions 5 for 16 subjects } \\
\hline & Out.side (\%) & In.side (\%) \\
\hline 1 & 44 & 56 \\
\hline 2 & 11 & 89 \\
\hline 3 & 12 & 88 \\
\hline 4 & 44 & 56 \\
\hline 5 & 59 & 41 \\
\hline 6 & 50 & 50 \\
\hline 7 & 50 & 50 \\
\hline 8 & 47 & 53 \\
\hline 9 & 41 & 59 \\
\hline 10 & 27 & 73 \\
\hline 11 & 36 & 64 \\
\hline 12 & 15 & 85 \\
\hline 13 & 9 & 91 \\
\hline 14 & 39 & 61 \\
\hline 15 & 49 & 51 \\
\hline 16 & 60 & 40 \\
\hline Average & $37.06 \pm 0.01$ & $62.93 \pm 0.01$ \\
\hline
\end{tabular}


The percentage for good feedback (1) present in.side (to target), the percentage for bad feedback $(0)$ present out.side (out target), note from table (3), the percentage and average for good feedback (1) high compare with percentage and average for bad feedback (0)except subject (16) notes the conversely, with an error ratio its (0.01 in session 5).

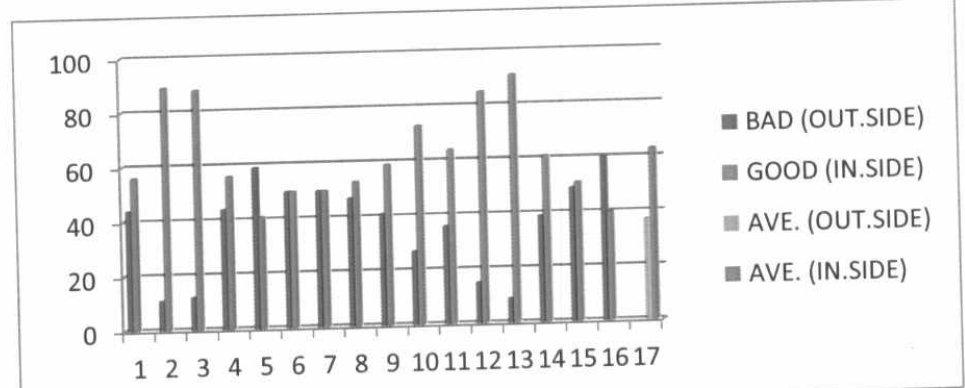

Figure 8: the chart table explains in.side, out.side, average in.side, and average out.side for session 5 , note the subjects $(6$ \& 7) percentage for feedback $(0$ and 1$)$ its equal.

\subsection{Performance of each electrode:}

The human brain divided into several lobes its: Frontal denote is $(\mathrm{F})$, Temporal denote is $(\mathrm{T})$, Central denote is (C), Parietal denote is (P), and Occipital denote is $(\mathrm{O})$, for each lobe specific functions. Shown Figure 9 explain the brain lobes and function of each lobe [14].

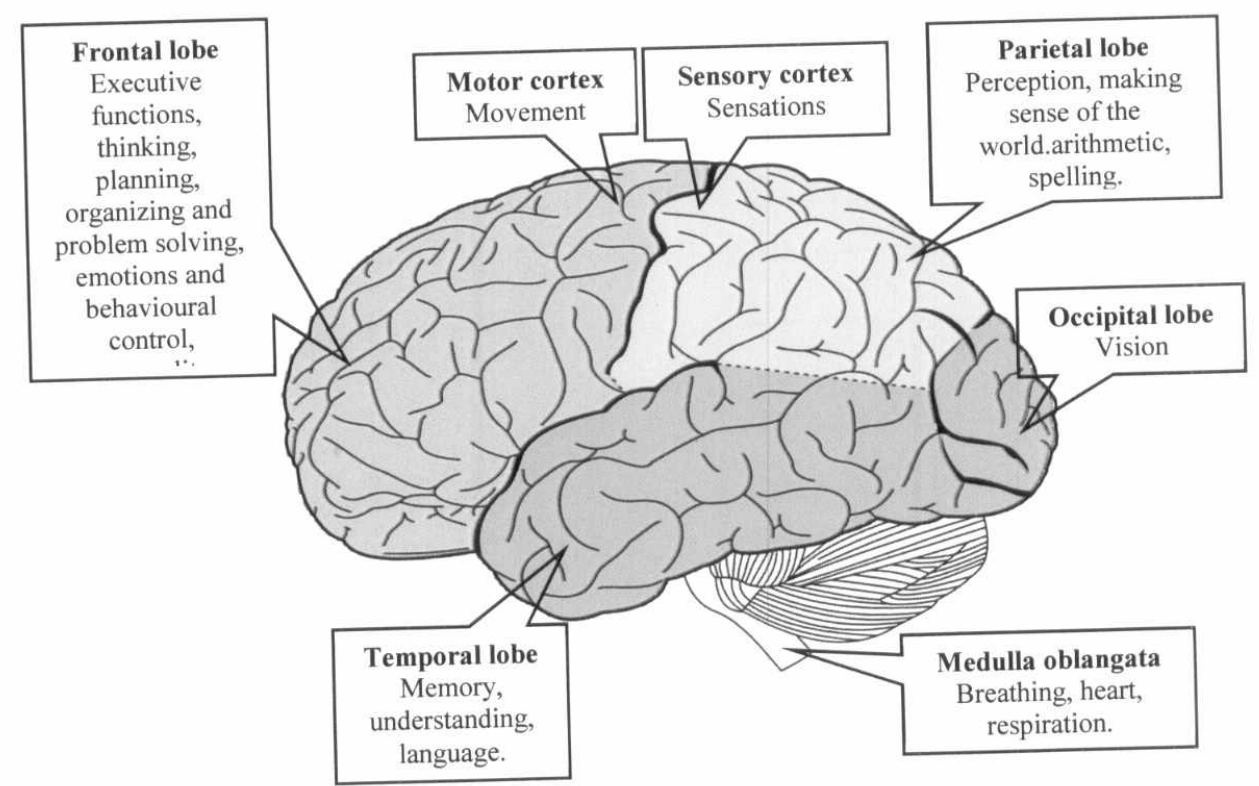

Figure 9 brain lobes and function for each lobe [14] 
Notes by form Figure 9, the central-parietal lobe (P) its responsible on the spelling task, typically done recorded P300 signal from centro-parietal electrodes, therefore the electrodes its placed on the lobe (P) its responsible on the speller task, and correlated to this task, based on 10-20 system international.

Using independent component analysis (ICA) is a statistical procedure that splits a set of mixed signals figures $(10-25)$, explain stimulus armation on the nature of the signal, using for actifact removal, the then bad feedback (negative), if

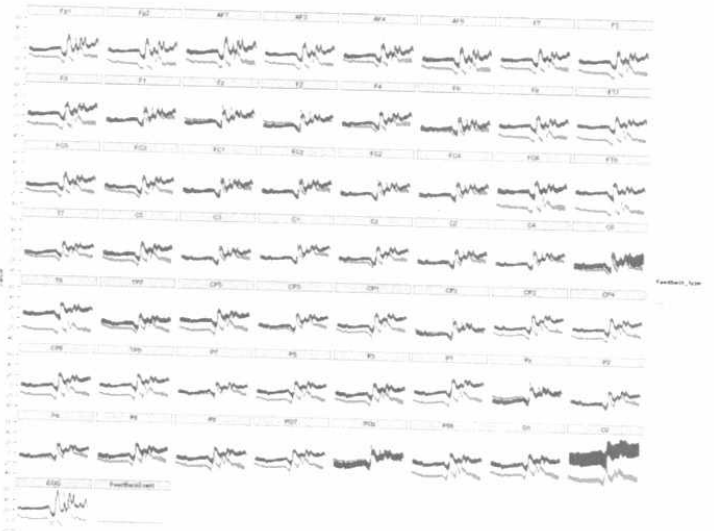

Figure 10: Show feedback type, if value $(0)$ then bad feedback, if value (1) good feedback, stimulus average for subject 1 for all sessions.

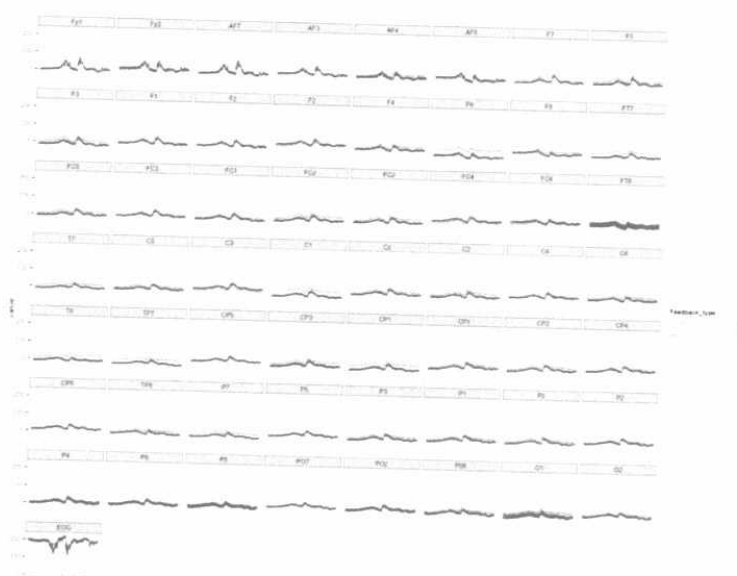

Figure 12: Show feedback type, if value $(0)$ then bad feedback, if value (1) good feedback, stimulus average
for subject 3 for all sessions.

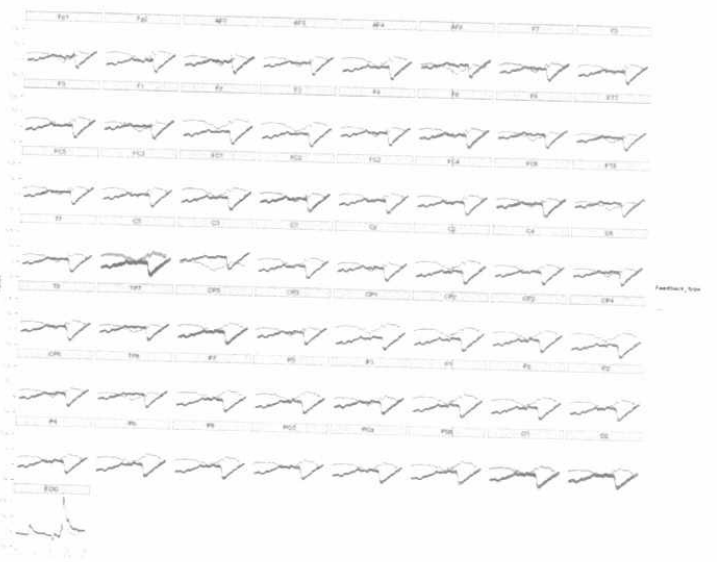

Figure 11: Show feedback type, if value $(0)$ then bad feedback, if value (1) good feedback, stimulus average for subject 2 for all sessions.

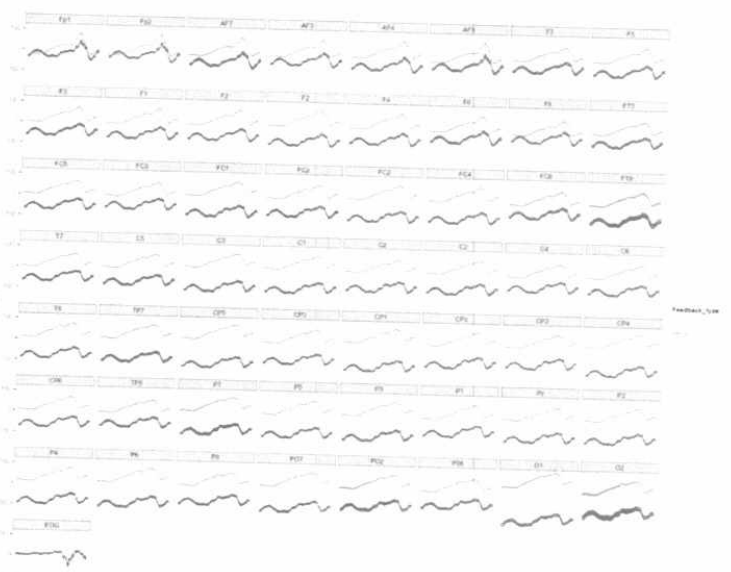

Figure 13: Show feedback type, if value $(0)$ then bad feedback, if value (1) good feedback, stimulus average for subject 4 for all sessions. 


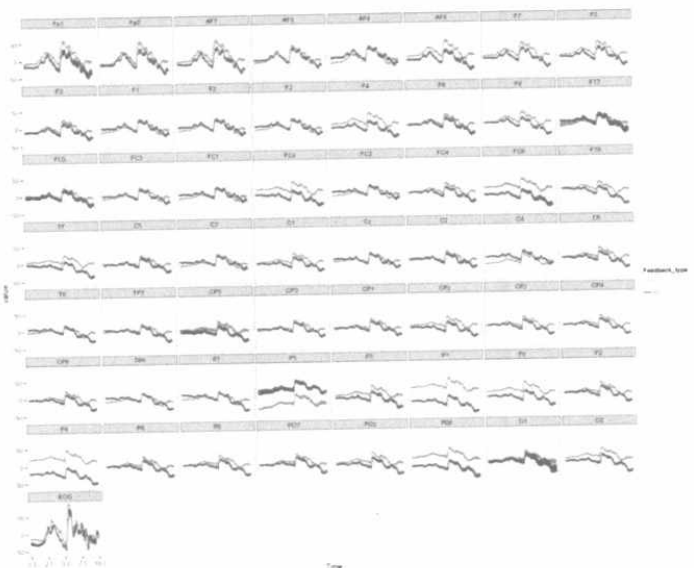

Figure 14: Show feedback type, if value ( 0 ) then bad feedback, if value (1) good feedback, stimulus average for subject 5 for all sessions.

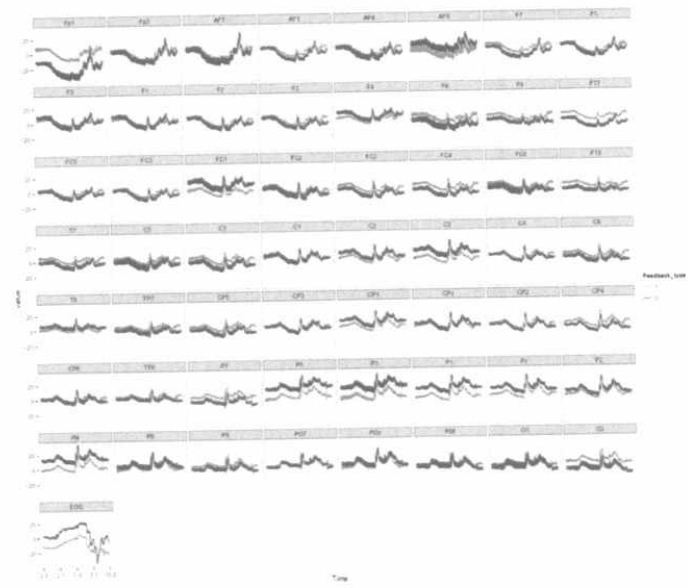

Figure 16: Show feedback type, if value (0) then bad feedback, if value (1) good feedback, stimulus average for subject 7 for all sessions.

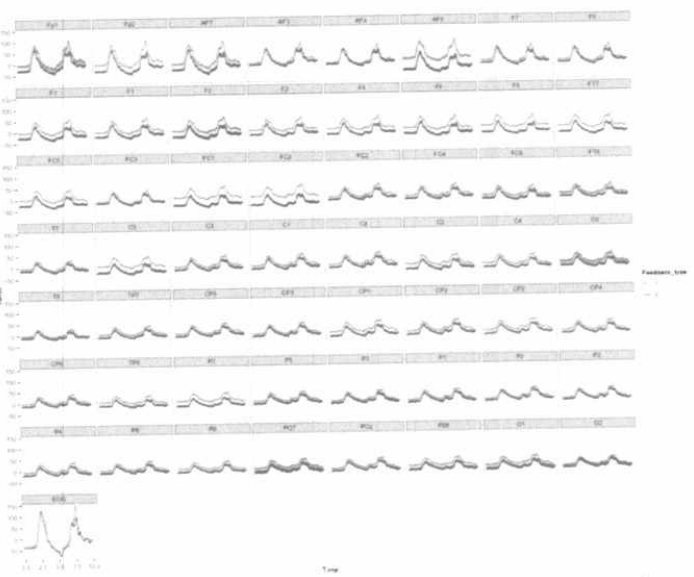

Figure 15: Show feedback type, if value ( 0 ) then bad feedback, if value (1) good feedback, stimulus average for subject 6 for all sessions.

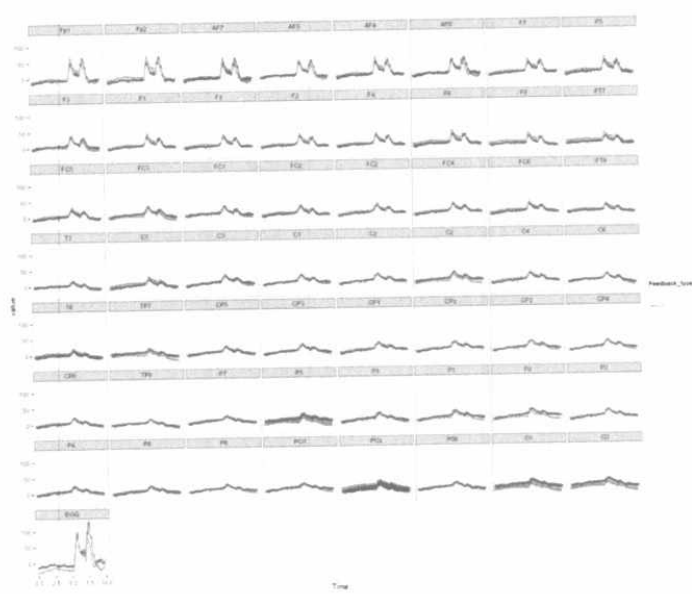

Figure 17: Show feedback type, if value (0) then bad feedback, if value (1) good feedback, stimulus average for subject 8 for all sessions. 


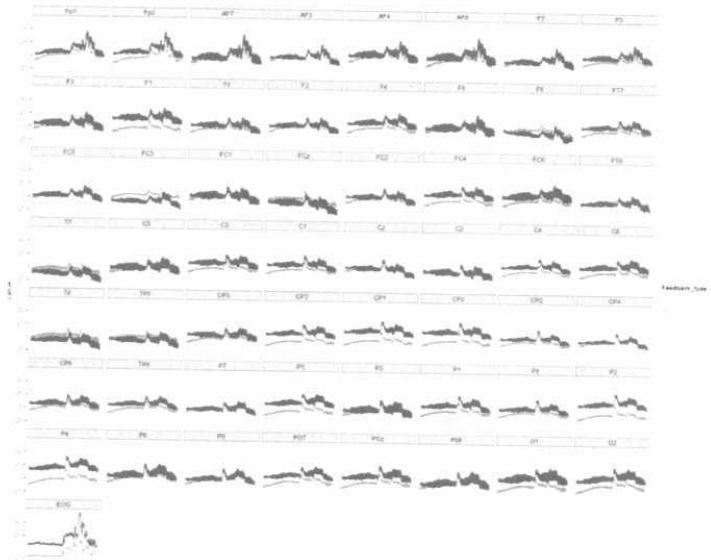

Figure 18: Show feedback type, if value $(0)$ then bad feedback, if value (1) good feedback, stimulus average for subject 9 for all sessions.

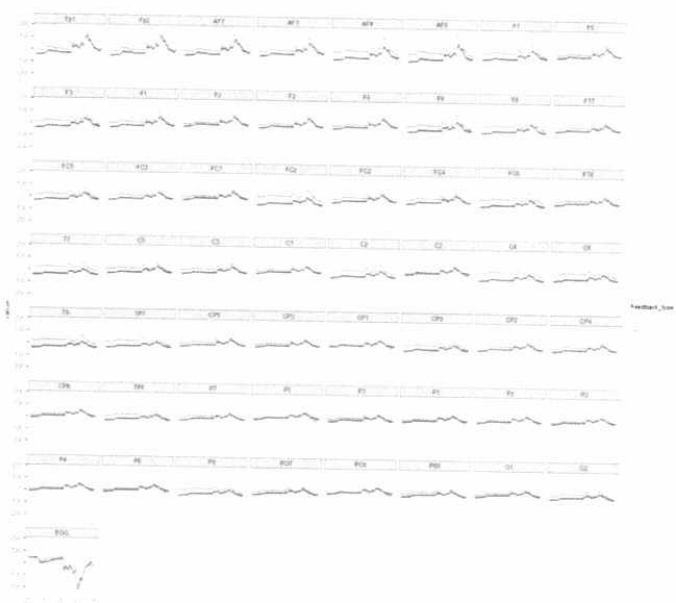

Figure 20: Show feedback type, if value $(0)$ then bad feedback, if value (1) good feedback, stimulus average for subject 11 for all sessions.

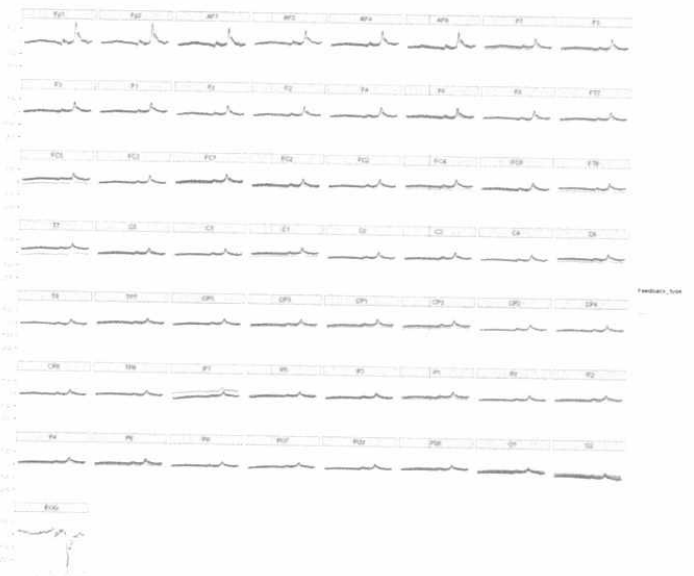

Figure 19: Show feedback type, if value (0) then bad feedback, if value (1) good feedback, stimulus average for subject 10 for all sessions.

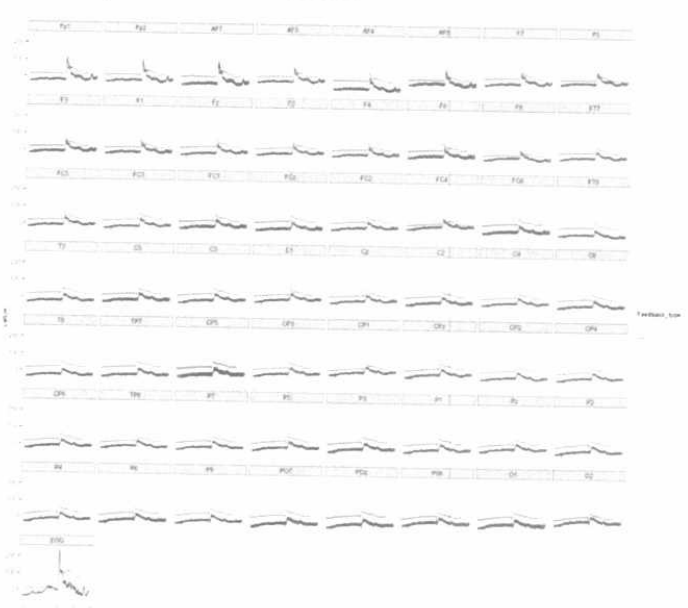

Figure 21: Show feedback type, if value $(0)$ then bad feedback, if value (1) good feedback, stimulus average for subject 12 for all sessions. 


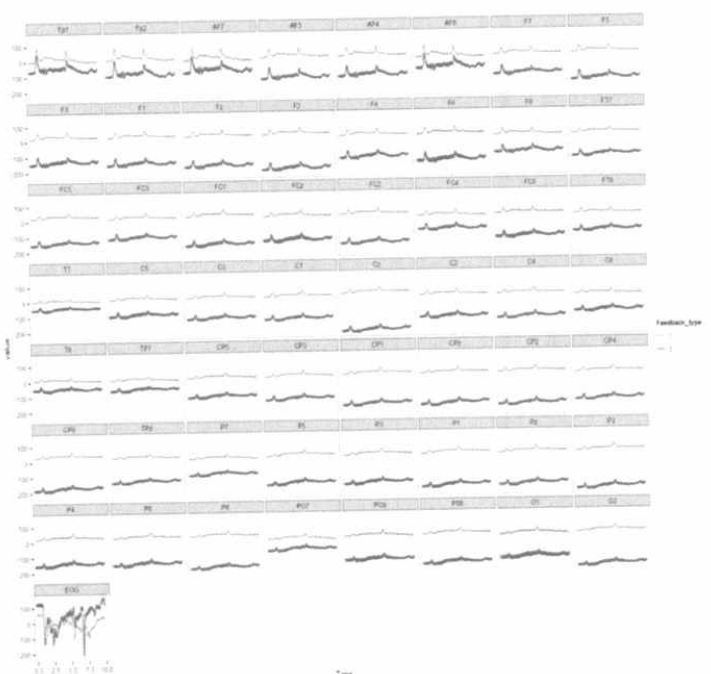

Figure 22: Show feedback type, if value ( 0 ) then bad feedback, if value (1) good feedback, stimulus average for subject 13 for all sessions.

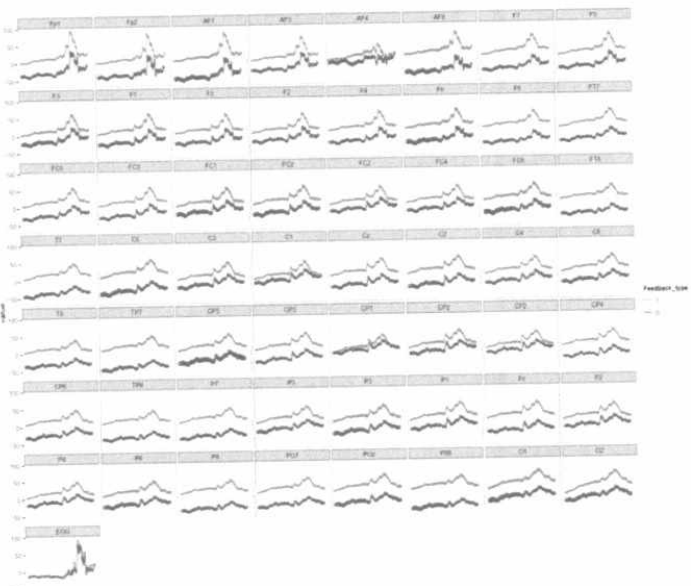

Figure 24: Show feedback type, if value ( 0 ) then bad feedback, if value (1) good feedback, stimulus average for subject 15 for all sessions.

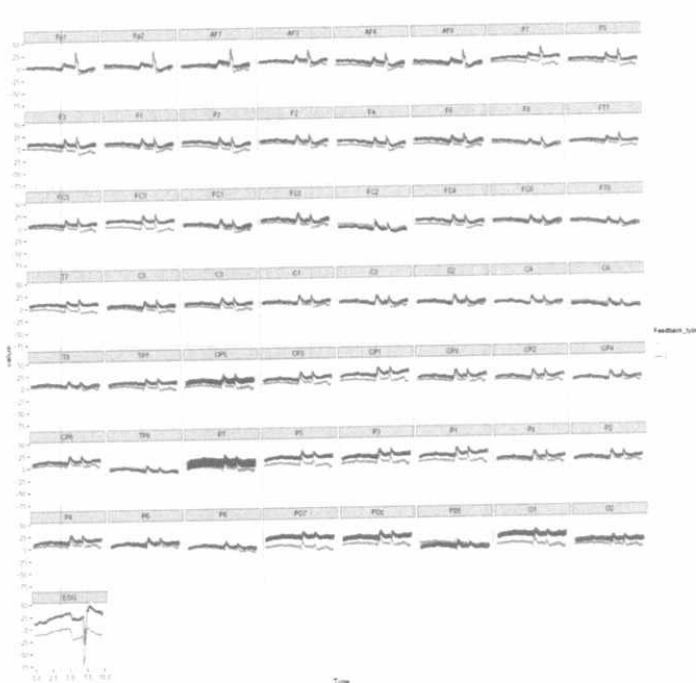

Figure 23: Show feedback type, if value $(0)$ then bad feedback, if value (1) good feedback, stimulus average for subject 14 for all sessions.

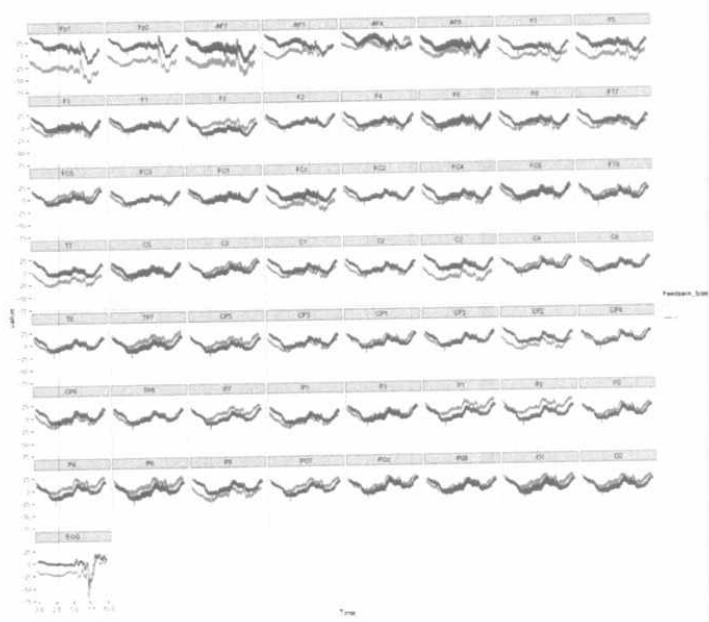

Figure 25: Show feedback type, if value (0) then bad feedback, if value (1) good feedback, stimulus average for subject 16 for all sessions. 


\subsection{Summary of results:}

5.1.1. Computing numbers of feedback ( 0 or 1$)$ for all subjects and all sessions, explaining in table 4 . Table 4: Explain numbers of feedback (bad (0) or good (1)), for each sessions for all subjects.

\begin{tabular}{|c|c|c|c|c|c|c|c|c|c|c|}
\hline \multirow[b]{2}{*}{ Subjects } & \multicolumn{2}{|c|}{ Session 1} & \multicolumn{2}{|c|}{ Session 2} & \multicolumn{2}{|c|}{ Session 3} & \multicolumn{2}{|c|}{ Session 4} & \multicolumn{2}{|c|}{ Session 5} \\
\hline & $\begin{array}{c}\text { Bad } \\
(0)\end{array}$ & $\begin{array}{c}\text { Good } \\
\text { (1) }\end{array}$ & Bad (0) & $\begin{array}{c}\text { Good } \\
(1) \\
\end{array}$ & Bad (0) & $\begin{array}{c}\text { Good } \\
\text { (1) }\end{array}$ & $\operatorname{Bad}(0)$ & $\begin{array}{l}\text { Good } \\
\text { (1) }\end{array}$ & Bad (0) & $\begin{array}{c}\text { Good } \\
\text { (1) }\end{array}$ \\
\hline 1 & 10 & 50 & 18 & 42 & 24 & 36 & 25 & 35 & 44 & 56 \\
\hline 2 & 1 & 59 & 3 & 57 & 5 & 55 & 4 & 56 & 11 & 89 \\
\hline 3 & 4 & 56 & 7 & 53 & 3 & 57 & 7 & 53 & 12 & 88 \\
\hline 4 & 14 & 46 & 18 & 42 & 18 & 42 & 21 & 39 & 44 & 56 \\
\hline 5 & 19 & 41 & 19 & 41 & 27 & 33 & 27 & 33 & 59 & 41 \\
\hline 6 & 22 & 38 & 30 & 30 & 36 & 24 & 25 & 35 & 50 & 50 \\
\hline 7 & 23 & 37 & 16 & 44 & 18 & 42 & 18 & 42 & 50 & 50 \\
\hline 8 & 16 & 44 & 18 & 42 & 22 & 38 & 25 & 35 & 47 & 53 \\
\hline 9 & 6 & 54 & 17 & 43 & 24 & 36 & 26 & 34 & 41 & 59 \\
\hline 10 & 9 & 51 & 14 & 46 & 14 & 46 & 15 & 45 & 27 & 73 \\
\hline 11 & 17 & 43 & 14 & 46 & 18 & 42 & 19 & 41 & 36 & 64 \\
\hline 12 & 2 & 58 & 2 & 58 & 3 & 57 & 5 & 55 & 15 & 85 \\
\hline 13 & 3 & 57 & 4 & 56 & 6 & 54 & 5 & 55 & 9 & 91 \\
\hline 14 & 18 & 42 & 24 & 36 & 21 & 39 & 17 & 43 & 39 & 61 \\
\hline 15 & 6 & 54 & 9 & 51 & 14 & 46 & 23 & 37 & 49 & 51 \\
\hline 16 & 25 & 35 & 23 & 37 & 30 & 30 & 21 & 39 & 60 & 40 \\
\hline
\end{tabular}

For each session (60) feedback (0 or 1), except last session (fifth 100 feedback (0 or 1$)$ ) for all subjects, and all sessions. 5.1.2. Computing average between feedback ( 0 and 1) for all subjects and all sessions, through the
following equation:

$$
\text { Ave. }=\frac{\text { Sum of feedback }(0 \& 1)}{\text { Total of numbers for feedback }(0 \& 1)}
$$

This is an equation for each subject in fifth of sessions, table 5 explaining the average for feedback.

Table 5: Explain average of feedback ( 0 and 1$)$, for all sessions, and all subjects.

\begin{tabular}{|c|c|c|c|c|c|}
\hline \multirow{2}{*}{ Subjects } & \multicolumn{5}{|c|}{ Average of feedback types for all sessions and all subjects } \\
\cline { 2 - 6 } & Session 1 & Session 2 & Session 3 & Session 4 & Session 5 \\
\hline $\mathbf{1}$ & 0.83 & 0.7 & 0.6 & 0.58 & 0.56 \\
\hline $\mathbf{2}$ & 0.98 & 0.95 & 0.91 & 0.93 & 0.89 \\
\hline $\mathbf{3}$ & 0.93 & 0.88 & 0.95 & 0.88 & 0.88 \\
\hline $\mathbf{4}$ & 0.76 & 0.7 & 0.7 & 0.65 & 0.56 \\
\hline $\mathbf{5}$ & 0.68 & 0.68 & 0.55 & 0.55 & 0.41 \\
\hline $\mathbf{6}$ & 0.63 & 0.5 & 0.4 & 0.58 & 0.5 \\
\hline $\mathbf{7}$ & 0.61 & 0.73 & 0.7 & 0.7 & 0.5 \\
\hline $\mathbf{8}$ & 0.73 & 0.7 & 0.63 & 0.75 & 0.53 \\
\hline $\mathbf{9}$ & 0.9 & 0.71 & 0.6 & 0.56 & 0.59 \\
\hline $\mathbf{1 0}$ & 0.85 & 0.76 & 0.76 & 0.75 & 0.73 \\
\hline $\mathbf{1 1}$ & 0.71 & 0.76 & 0.7 & 0.68 & 0.64 \\
\hline $\mathbf{1 2}$ & 0.96 & 0.96 & 0.95 & 0.91 & 0.85 \\
\hline $\mathbf{1 3}$ & 0.95 & 0.93 & 0.9 & 0.91 & 0.91 \\
\hline $\mathbf{1 4}$ & 0.7 & 0.6 & 0.65 & 0.71 & 0.61 \\
\hline $\mathbf{1 5}$ & 0.9 & 0.85 & 0.76 & 0.61 & 0.51 \\
\hline $\mathbf{1 6}$ & 0.58 & 0.61 & 0.5 & 0.65 & 0.4 \\
\hline
\end{tabular}


5.1.3. As previously passed, from where number of feedback for each subject, in all sessions, the table 6 listing sequences of feedback for all subjects and all sessions.

Table 6: Explain the sequence of feedback for each subject in all sessions.

\begin{tabular}{|c|c|c|}
\hline \multirow{5}{*}{ Subject 1} & Session 1 & 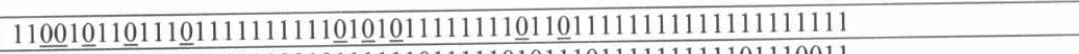 \\
\hline & Session 2 & $11 \underline{000} 1 \underline{0} 1100100111110010111111 \underline{0} 11111 \underline{0} 1 \underline{0} 111 \underline{0} 1111111111 \underline{0} 1110011$ \\
\hline & Session 3 & $1111111 \underline{0} 1 \underline{0} 111111 \underline{0} 11001011 \underline{0} 111 \underline{0} 1000010110011101100101011000$ \\
\hline & Session 4 & $111 \underline{00} 111 \underline{0} 1 \underline{0} 111 \underline{0} 1 \underline{0} 11 \underline{0} 1111 \underline{00} 1 \underline{0} 1 \underline{00} 1 \underline{0} 1 \underline{0} 111 \underline{00} 1 \underline{0} 1 \underline{0} 1 \underline{00} 11 \underline{0} 111 \underline{00} 1 \underline{0} 11$ \\
\hline & Session 5 & 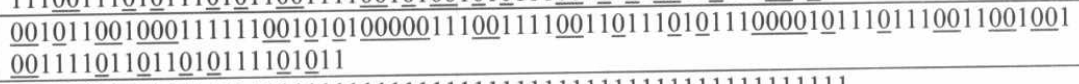 \\
\hline \multirow{5}{*}{ Subject 2} & Session 1 & $111 \underline{0} 1111111111111111111111111111111111111111111111111111111$ \\
\hline & Session 2 & $111111111111 \underline{0} 1111111111 \underline{0} 111111111111111111111111 \underline{\underline{0} 111111111}$ \\
\hline & Session 3 & $111 \underline{0} 1111111111 \underline{00} 111111111111111111111111111111111111 \underline{0} 111111 \underline{0}$ \\
\hline & Session 4 & $11111111 \underline{\underline{0}} 111111111111111111111111111111111 \underline{\underline{0}} 1111 \underline{\underline{0} 01111111111}$ \\
\hline & Session 5 & $\begin{array}{l}1111111111111 \underline{0} 11111111111111 \underline{0} 11111111111 \underline{0} 1 \underline{00} 1 \underline{0} 1111111111111111 \underline{0} 1111111 \underline{0} 111 \\
\underline{0} 11111111111 \underline{\underline{0}} 111 \underline{\underline{0}} 11111\end{array}$ \\
\hline \multirow{5}{*}{ Subject 3} & Session 1 & 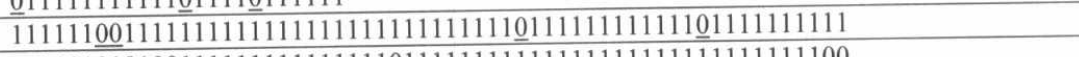 \\
\hline & Session 2 & $11111 \underline{0} 01 \underline{0} 1 \underline{00} 111111111111111 \underline{0} 11111111111111111111111111111100$ \\
\hline & Session 3 & 1011111111111111110111111111111111111111111111111011111111111 \\
\hline & Session 4 & $111 \underline{0} 1111111111111111110 \underline{0} 111 \underline{0} 1 \underline{0} 1111111111111111111 \underline{0} 1111111 \underline{00}$ \\
\hline & Session 5 & 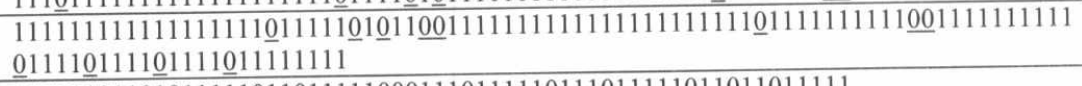 \\
\hline \multirow{4}{*}{ Subject 4} & Session 1 & 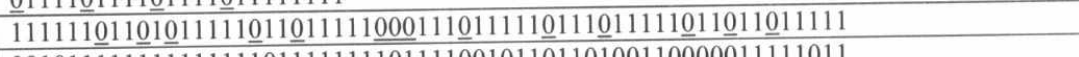 \\
\hline & Session 2 & $\underline{00} 1 \underline{0} 11111111111111 \underline{0} 1111111 \underline{0} 1111 \underline{00} 1 \underline{0} 11 \underline{0} 11 \underline{\underline{0}} 1 \underline{00} 110000011111 \underline{0} 11$ \\
\hline & Session 3 & $1111110000111111 \underline{0} 1 \underline{0} 100011111111 \underline{0} 1111 \underline{0} 111 \underline{0} 1111111110011100010$ \\
\hline & Session 4 & $\underline{0} 1 \underline{0} 11 \underline{00} 1 \underline{0} 11 \underline{0} 11111 \underline{00} 1 \underline{0} 111111 \underline{0} 1 \underline{\underline{p}} 1111111 \underline{0} 1001100110001010111111$ \\
\hline \multirow{4}{*}{ Subject 5} & $\begin{array}{l}\text { Session } 1 \\
\text { Session } 2\end{array}$ & 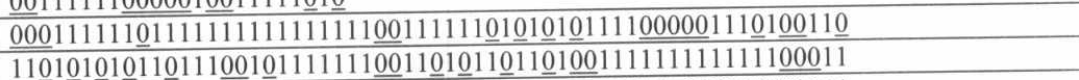 \\
\hline & Session 3 & 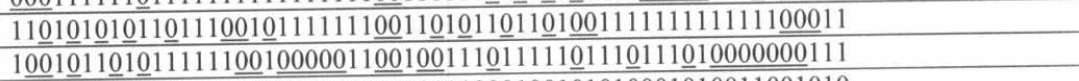 \\
\hline & Session 4 & 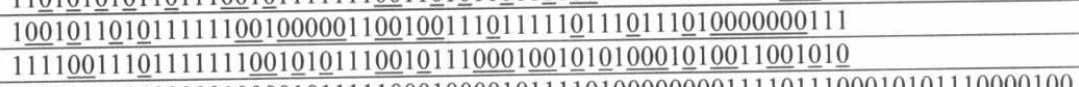 \\
\hline & Session 5 & 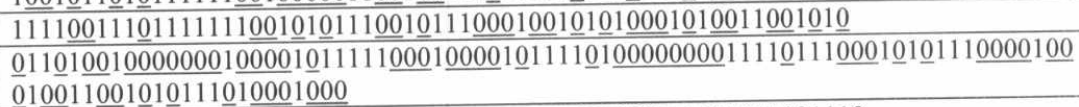 \\
\hline \multirow{5}{*}{ Subject 6} & Session 1 & $\underline{0} 1 \underline{00} 1 \underline{00} 1 \underline{0} 1 \underline{0} 111 \underline{0} 10001000$ \\
\hline & $\begin{array}{l}\text { Session } 2 \\
\text { Session } 3\end{array}$ & 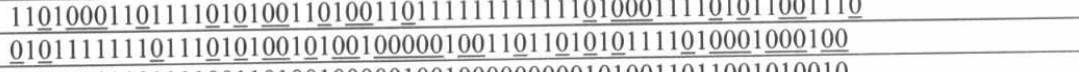 \\
\hline & $\begin{array}{l}\text { Session } 3 \\
\text { Session } 4\end{array}$ & $1 \underline{0} 1 \underline{0} 11 \underline{0} 11 \underline{0} 1111 \underline{00} 11 \underline{0} 10010000010010000000001010011011001010010$ \\
\hline & & $1 \underline{0} 1 \underline{0} 1 \underline{0} 11 \underline{0} 11 \underline{0} 1 \underline{0} 100000110011111111100000101111111 \underline{00} 1 \underline{0} 111 \underline{0} 1111 \underline{00}$ \\
\hline & $\begin{array}{l}\text { Session } 5 \\
\text { Session } 1\end{array}$ & 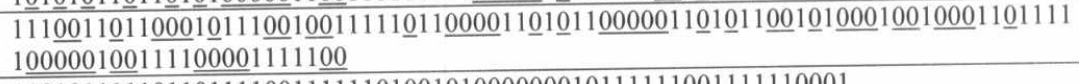 \\
\hline \multirow{4}{*}{ Subject 7} & $\begin{array}{l}\text { Session } 1 \\
\text { Session } 2\end{array}$ & $11 \underline{0} \underline{0} 11111 \underline{0} 11 \underline{0} 111100111111 \underline{0} 10010100000001011111100111111 \underline{000} 1$ \\
\hline & $\begin{array}{l}\text { Session } 2 \\
\text { Session } 3 \\
\end{array}$ & 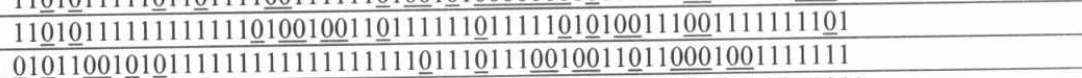 \\
\hline & Session 4 & $\underline{0} 1 \underline{0} 11001 \underline{0} 1 \underline{0} 11111111111111111 \underline{0} 111 \underline{0} 111 \underline{00} 1 \underline{00} 11 \underline{0} 11 \underline{000} 1 \underline{00} 1111111$ \\
\hline & Session 5 & 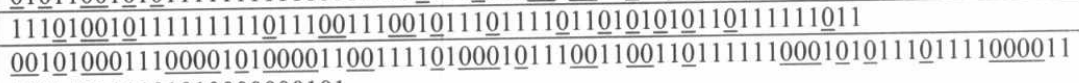 \\
\hline \multirow{5}{*}{ Subject 8} & Session 1 & 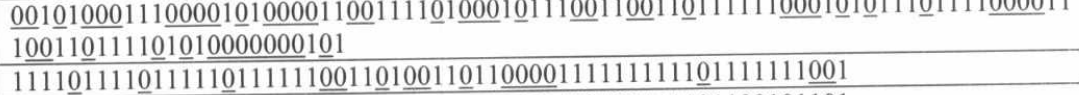 \\
\hline & Session 2 & 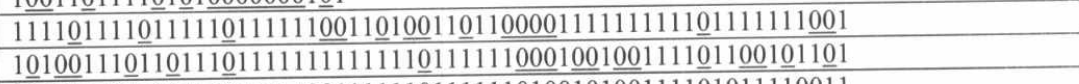 \\
\hline & Session 3 & 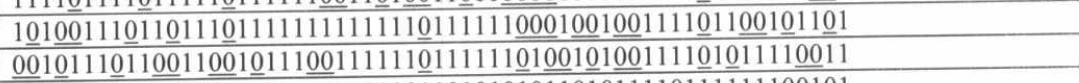 \\
\hline & Session 4 & 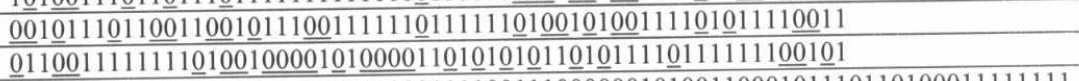 \\
\hline & Session 5 & 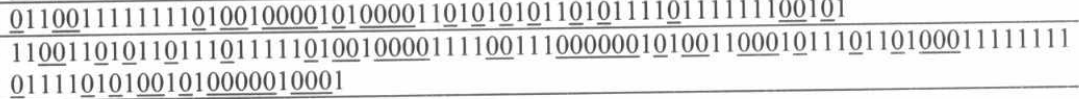 \\
\hline
\end{tabular}




\begin{tabular}{|c|c|c|}
\hline \multirow{5}{*}{ Subject 9} & Session 1 & $\underline{0} 11111111111111111111111111 \underline{01} 11111111111111111 \underline{\underline{0}} 11 \underline{\underline{00}} 11111 \underline{\underline{0}} 111$ \\
\hline & Session 2 & $11111111 \underline{0} 1111 \underline{\underline{0}} 11 \underline{0} 1111 \underline{\underline{0}} 111111 \underline{00001} \underline{0} 11 \underline{00} 1 \underline{0} 1111111111 \underline{000110111 \underline{0}}$ \\
\hline & Session 3 & $111 \underline{0} 11 \underline{0111} 0111 \underline{0} 1 \underline{0011} \underline{0} 1 \underline{00} 10001 \underline{0} 1 \underline{0} 11 \underline{0} 11 \underline{0} 1111 \underline{0} 111111 \underline{001} 1 \underline{0} 1 \underline{0} 1 \underline{00} 1 \underline{0}$ \\
\hline & Session 4 & $1111111 \underline{0} 11110011101000011100010000010111001000111111 \underline{0} 1 \underline{0} 1 \underline{0} 1 \underline{0} 1$ \\
\hline & Session 5 & 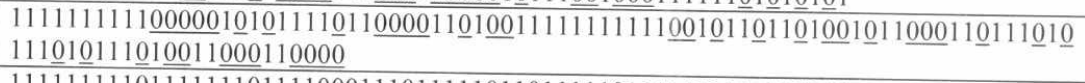 \\
\hline \multirow{5}{*}{$\begin{array}{l}\text { Subject } \\
10\end{array}$} & Session 1 & 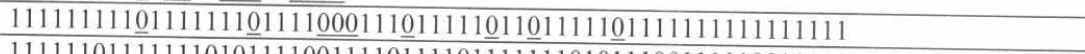 \\
\hline & Session 2 & $111111 \underline{0} 1111111 \underline{0} 1 \underline{0} 1111 \underline{00} 1111 \underline{0} 1111 \underline{0} 1111111 \underline{0} \underline{0} 1110011111 \underline{001111}$ \\
\hline & Session 3 & 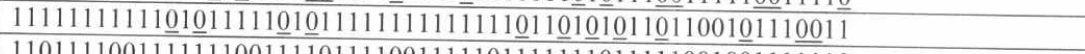 \\
\hline & Session 4 & 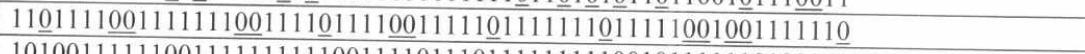 \\
\hline & Session 5 & 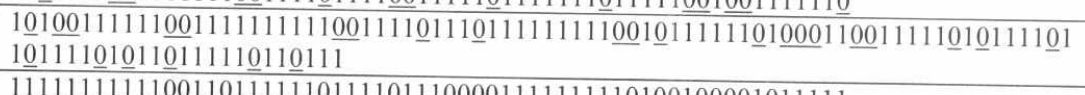 \\
\hline \multirow{5}{*}{$\begin{array}{l}\text { Subject } \\
11\end{array}$} & Session 1 & 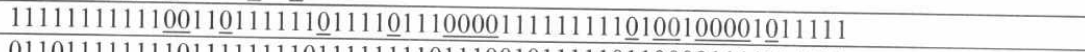 \\
\hline & Session 2 & 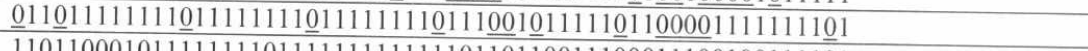 \\
\hline & Session 3 & $11 \underline{0} 110001 \underline{0} 11111111 \underline{0} 111111111111 \underline{\underline{0}} 11 \underline{0} 1100111000111001001111 \underline{0} 1$ \\
\hline & Session 4 & 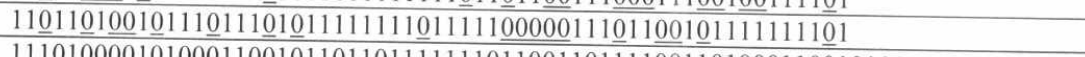 \\
\hline & Session 5 & $\begin{array}{l}111 \underline{0} 1000010100011 \underline{00} 1 \underline{0} 11 \underline{0} 11 \underline{0} 1111111 \underline{0} 11 \underline{00} 11 \underline{0} 1111 \underline{00} 11 \underline{0} 1 \underline{000} 11 \underline{00} 1 \underline{0} 111111 \underline{0} 1 \underline{0} 11 \underline{0} 111 \\
111 \underline{00} 11 \underline{0} 1 \underline{0} 1 \underline{0} 11 \underline{0} 111111111\end{array}$ \\
\hline \multirow{5}{*}{$\begin{array}{l}\text { Subject } \\
12\end{array}$} & Session 1 & 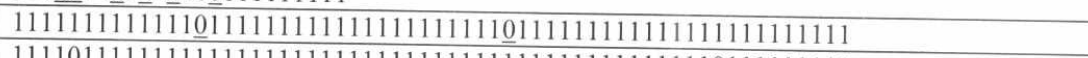 \\
\hline & Session 2 & $1111 \underline{0} 11111111111111111111111111111111111111111111 \underline{0111111111}$ \\
\hline & Session 3 & $1111111111111 \underline{00} 1111111111111 \underline{0} 111111111111111111111111 \underline{0} 111111$ \\
\hline & Session 4 & $11111111111111111111111 \underline{0} 1 \underline{0} 11111111111111111 \underline{0} 111 \underline{0} 1111111 \underline{0} 111$ \\
\hline & Session 5 & $\begin{array}{l}11 \underline{0} 11 \underline{0} 1111111111111111 \underline{\underline{0}} 1 \underline{0} 111111111 \underline{00} 1111111111111 \underline{0} 111 \underline{0} 11111 \underline{0} 1111111 \underline{0} 11 \underline{0} 11 \underline{0} \\
\underline{0} 1111111111111 \underline{0} 11111 \underline{0} 11\end{array}$ \\
\hline \multirow{5}{*}{$\begin{array}{l}\text { Subject } \\
13\end{array}$} & Session 1 & 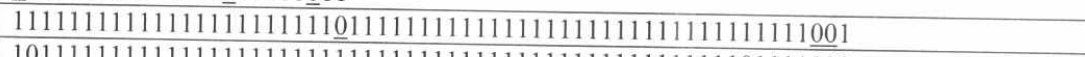 \\
\hline & Session 2 & $1 \underline{\underline{0}} 1111111111111111111111111111111111111111111111111 \underline{011111 \underline{00}}$ \\
\hline & Session 3 & $1111111 \underline{0} 1111111111111111111111111111 \underline{0} 111111 \underline{0} 111 \underline{0} 1111 \underline{0} 1 \underline{011111}$ \\
\hline & Session 4 & $111 \underline{0} 11111111111111111 \underline{0} 111111111111111011111111111111 \underline{\underline{0}} 11111 \underline{\underline{0}}$ \\
\hline & Session 5 & $\begin{array}{l}1111111 \underline{0} 1111111111 \underline{01111} \underline{0} 111111111111111111111111111111111 \underline{\underline{0} 00} 111111111111111 \\
\underline{0} 1111111111111 \underline{00} 11 \underline{\underline{0}} 11111\end{array}$ \\
\hline \multirow{5}{*}{$\begin{array}{l}\text { Subject } \\
14\end{array}$} & Session 1 & 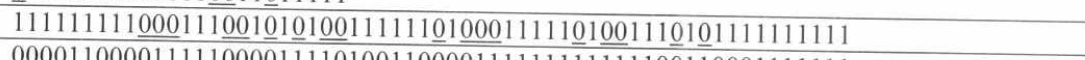 \\
\hline & Session 2 & 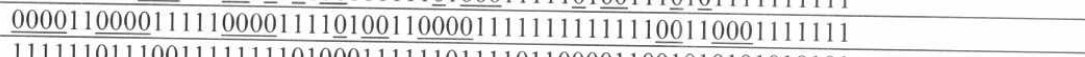 \\
\hline & Session 3 & $11111 \underline{0} 111001111111101000111111 \underline{0} 1111 \underline{01} 100001100101 \underline{0} 1 \underline{0} 1 \underline{0} 1 \underline{0} 1 \underline{0} 1$ \\
\hline & Session 4 & $1 \underline{0} 11111001100000111111 \underline{0} 111111 \underline{\underline{0}} 1 \underline{0} 1110011 \underline{0} 11111111 \underline{0} 1 \underline{0} 1111 \underline{\underline{0}} 11$ \\
\hline & Session 5 & $\begin{array}{l}11110001011000011110010111111 \underline{0} 1 \underline{00} 1100001001111 \underline{0} 11 \underline{0} 1 \underline{0} 1111 \underline{0} 1 \underline{0} 1000111 \underline{0} 111111111 \\
1 \underline{000011} 11001111 \underline{0} 1 \underline{0} 1 \underline{0} 11 \underline{0}\end{array}$ \\
\hline \multirow{5}{*}{$\begin{array}{l}\text { Subject } \\
15\end{array}$} & Session 1 & $1111111111111111 \underline{00} 1111111111 \underline{\underline{0}} 1 \underline{00} 11111111111 \underline{\underline{0}} 1111111111111111$ \\
\hline & Session 2 & $1111111 \underline{0} 11111111111111111111 \underline{\underline{0}} 1 \underline{\underline{0}} 111 \underline{0} 11 \underline{0} 11 \underline{0} 111111 \underline{\underline{0}} 111 \underline{00111111}$ \\
\hline & Session 3 & $\underline{0} 11111111111 \underline{0} 111111111111111 \underline{\underline{0}} 1 \underline{\underline{0}} 1 \underline{\underline{0}} 11 \underline{0} 1111000001111111 \underline{00011111}$ \\
\hline & Session 4 & $1111 \underline{0} 10101010111010011001011001011 \underline{0} 111 \underline{0} 111 \underline{0} 1 \underline{0} 110001011011111$ \\
\hline & Session 5 & 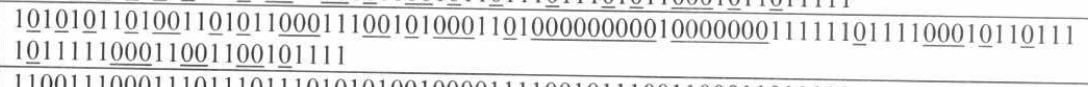 \\
\hline \multirow{5}{*}{$\begin{array}{l}\text { Subject } \\
16\end{array}$} & Session 1 & 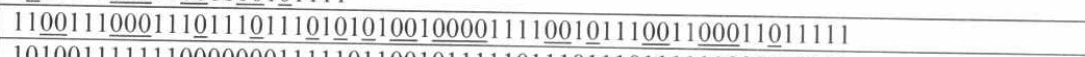 \\
\hline & Session 2 & $1 \underline{0} 1001111111000000011111011001011111011101110111111000110001$ \\
\hline & Session 3 & 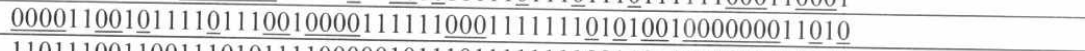 \\
\hline & Session 4 & $11 \underline{0} 111001100111 \underline{0} 1 \underline{0} 111100000101110111111110011011010010111111$ \\
\hline & Session 5 & $\underline{1001} 1 \underline{00} 100001 \underline{0000} 1 \underline{000} 11 \underline{00} 1 \underline{00} 1 \underline{0} 1 \underline{000} 11 \underline{0000} 1 \underline{0} 111 \underline{00} 1 \underline{0} 1 \underline{0} 1 \underline{00} 1 \underline{0} 111 \underline{0000000} 111 \underline{0} 1 \underline{00} 1 \underline{0} 1 \underline{0} 1 \underline{000}$ \\
\hline
\end{tabular}
Each subject (340) feedback ( 0 or 1$),(240)$ feedback for ( $1-4$ of sessions), (100) feedback for session
5 . 


\section{Future work:}

The following suggestion uses in the future studies for evaluation or development the P300 speller robust and accurate, its: the researches evaluation the speller matrix making the letters are large size and coloring, and background coloring or none, using slow and fast mode. Using P300 speller for Arabic letters. Detection and correction errors event during P300 speller task whether offline and online.

\section{Conclusion}

In this paper a new method for increase accuracy of classification for P300 speller, including: Feature extraction using (PCA) based on percentage for good feedback (1) and bad feedback (0), good feedback (in.side) and bad feedback (out.side), Classification using (SVML) for computing numbers of feedback whether ( 0 or 1$)$ for all subjects and all sessions, sequences of feedback ( 0 or 1$)$ for all subjects and all sessions, computing average between feedback ( 0 or 1$)$ for all subjects and all sessions. Finally calculated performance of each electrode using (ICA) whether correlated or uncorrelated speller task for all subjects and all sessions.

\section{References}

1. Luis Fernando Nicolas-Alonso, Jaime Gomez-Gil, "Brain Computer Interfaces, a Review", Sensors, 12, 1211-1279; doi:10.3390/s120201211, ISSN 1424-8220.

2. J. R. Wolpaw, N. Birbaumer, D. J. McFarland, G. Pfurtscheller, and T. M. Vaughan, "Braincomputer interfaces for communication and control." Clinical Neurophysiology, vol. 113, no. 6, pp. 767-791, 2002.

3. B. H. Dobkin, "Brain-computer interface technology as a tool to augment plasticity and outcomes for neurological rehabilitation," Journal of Physiology, vol. 579, part 3, pp. 637-642, 2007.

4. J. D. Millan, R. Rupp, G. R. Muller-Putz et al. et al., "Combining brain-computer interfaces and assistive technologies: state-of-the-art and challenges," Frontiers in Neuroscience, vol. 4, article 161, 2010.

5. L. A. Farwell and E. Donchin, "Talking off the top of your head: toward a mental prosthesis utilizing event-related brain potentials," Electroencephalography and Clinical Neurophysiology, vol. 70, no. 6, pp. 510-523, 1988.

6. M. Fabiani, D. Karis, and E. Donchin, "P300 and recall in an incidental memory paradigm" Psychophysiology, vol. 23, no. 3, pp. 298-308, 1986.

7. E. Donchin, K. M. Spencer, and R. Wijesinghe, "The mental prosthesis: assessing the speed of a P300-based brain- computer interface," IEEE Transactions on Rehabilitation Engineering, vol. 8, no. 2, pp. 174-179, 2000.

8. Perrin Margaux, MabyEmmanue, DaligaultS'ebastien, Bertrand Olivier, and MattoutJ'er'emie, "Objective and Subjective Evaluation of Online Error Correction during P300-Based Spelling", Hindawi Publishing Corporation, Advances in Human-Computer Interaction, doi: $10.1155 / 2012 / 578295$.

9. Deepesh Kumar, "P300 detection for brain computer interface", master thesis, Department of Electronics \& Communication EngineeringNational Institute of Technology, Rourkela-769008, India, 2013.

10. Benjamin Blankertz, Christin Schäfer, Guido Dornhege, and Gabriel Curio, "Single Trial Detection of EEG Error Potentials: A Tool for Increasing BCI transmission rates", University Berlin, Berlin, Germany, 2014. 
11. Vanitha Narayan Raju, In-Ho Ra and Ravi Sankar, "A P300-Based BCI Classification Algorithm Using Least Square Support Vector Machine", International Journal of Software Engineering and Its Applications Vol. 9, No. 5, pp. 247-254, 2015.

12. Jonathan R. Wolpaw, Niels Birbaumer, Dennis J. McFarland, "Brain-computer interfaces for

13. Falkenstein, M., Hoormann, J., Christ, S., Hohnsbein, J. "ERP components on rean 2002. their functional significance: a tutorial". Biol. Psychol. 51, 87 components on reaction errors and

14. GuilhermeCarvalhalRibas, "The Cere 20121437, 2010. 